\title{
General Solutions of the Nonlinear PDEs Governing the Erosion Kinetics
}

\author{
D. E. PANAYOTOUNAKOS* and K. P. ZAFEIROPOULOS
}

Division of Mechanics, Department of Applied Sciences, National Technical University of Athens, Hellas

(Received 13 July 2001)

\begin{abstract}
We present the construction of the general solutions concerning the one-dimensional (1D) fully dynamic nonlinear partial differential equations (PDEs), for the erosion kinetics. After an uncoupling procedure of the above mentioned equations a second-order nonlinear PDE of the Monge type governing the porosity is derived, the general solution of which is constructed in the sense that a full complement of arbitrary functions (as many as the order) is introduced. Afterwards, we specify the above solution according to convenient initial conditions.
\end{abstract}

Key words: Erosion, kinetics, general solutions of nonlinear PDEs

\section{INTRODUCTION}

A review on sand production as a problem of petroleum engineering is given in the paper by Veeken, Davies, Kenter and Kooijman [1]. The production of solid models aim at optimizing the fluid production rates avoiding near-wellborn instabilities and damage of reservoir structure.

From the modeling point of view a basic mechanism, which is usually related to the sand production in oil-producing wells, is the following: Hydro-mechanical instabilities deriving from internal and surface erosion manifest themselves as releasing and transferring particles, mainly due to action of seepage forces in regions of fluid-pressure gradients. In the paper by Vardoulakis, Stavropoulou and Papanastasiou [2], the above aspect of sand production as a surface erosion phenomenon is studied. The approach in this paper is fully deterministic and is based on a three-phase mixture theory of a continuum consisting of skeleton solids, fluidized-solids and fluid. The erosion process is assumed to obey a constitutive law for particle transport originally proposed by Einstein [3] and later by Sakthivadivel and Irmay [4]. Two dimensionless full dynamic nonlinear PDEs for the erosion kinetics governing the porosity including the time function discharge are derived and numerical solutions through convenient initial or boundary conditions are given. In the present paper, based on [2], the decoupling of the above mentioned nonlinear system is presented and the formulation of a second-order nonlinear PDE of the Monge type concerning the porosity is derived. Afterwards, the general analytical solution of the porosity equation is constructed, in the sense that a full complement of arbitrary functions (as many as the order) is introduced.

\footnotetext{
* Corresponding author.
} 
We specify the above solution according to convenient initial conditions. Here we must point out that only special cases of nonlinear PDEs of the form $f\left(x, y, z, z_{x}, z_{y}, z_{x x}, z_{y y}, z_{x y}\right)=0$ have been integrated giving general solutions. A few of these, expressing general and important geometrical and physical problems, have been included by Forsyth [5] and Ames [6]. This is of little mathematical interest in this area, but hopefully the demand of engineering science will breathe new life into this subject, since the authors believe that the result from this area will lead to windows onto the nonlinear morass.

\section{MATHEMATICAL FORMULATION}

Consider a saturated porous granular medium consisting of solid, fluid and fluidized-solid particles. According to Vardoulakis, Stavropoulou and Papanastasiou [2] the set of mass balance equations of the erosion kinetics for a volume element of the medium are given by

$$
\begin{aligned}
& \frac{\partial \phi}{\partial t}=\frac{\dot{m}}{\rho_{\mathrm{s}}} \\
& \frac{\partial \phi}{\partial t}=\frac{\partial(c \phi)}{\partial t}+\frac{\partial\left(c q_{i}\right)}{\partial x}, \quad(i=1,2,3) \\
& \frac{\partial q_{i}}{\partial x_{i}}=0
\end{aligned}
$$

In these equations $\phi\left(x_{i}, t\right)$ denotes the porosity; $c\left(x_{i}, t\right)$ is the transport concentration of the fluidized-particles; $\dot{m}>0$ is the mass-generation term, which corresponds to the rate of mass eroded and fluidized at any time and point, while $q_{i}$ denotes the volume-discharge of mixture. Finally, $\rho_{\mathrm{s}}$ is the density of solids; $x_{i}$ denotes the location and $t$ the time. The above equations are not sufficient for the solution of the problem under consideration. Thus, this system must be enriched by a constitutive relation governing the filtration kinetics. Extensive experimental and theoretical studies in relation to the filtration of non-colloidal particles in porous media were run in the late 60 's by H.A. Einstein [3]. This work, summarized by Sakthivadivel and Irmay [4], resulted in a constitutive equation which governs the filtration kinetics. According to Vardoulakis, Stavropoulou and Papanastasiou [2] combination of the above mentioned ideas furnishes the following expression for the rate of eroded mass

$$
\dot{m}=\rho_{\mathrm{s}} \lambda(1-\phi) c\left\|q_{i}\right\|
$$

in which

$$
\lambda=(1-\varphi) \lambda^{\prime}
$$

The coefficient $\lambda^{\prime}$ has dimension of inverse length and must be determined experimentally. In the case of one dimensional erosion kinetics the Eqs. (1) and (2) are reduced to the simple ones

$$
\begin{aligned}
& \frac{\partial(c \phi)}{\partial t}=q \frac{\partial c}{\partial x}+\frac{\partial \phi}{\partial t} \\
& \frac{\partial \phi}{\partial t}=\lambda(1-\phi) c q, \\
& x \geq 0, \quad q(t)>0, \quad 0<\phi<1, \quad 0<c<1 .
\end{aligned}
$$


Finally, introducing in (3) the dimensionless quantities (see Ref. [2])

$$
\begin{aligned}
& X=\lambda x \Rightarrow \frac{\partial}{\partial x}=\lambda \frac{\partial}{\partial X}, \\
& T=\lambda \int_{0}^{t} q(\tau) \mathrm{d} \tau \Rightarrow \frac{\partial}{\partial t}=\lambda q \frac{\partial}{\partial T},
\end{aligned}
$$

we obtain the following nonlinear PDEs governing the erosion kinetics of the system under consideration

$$
\begin{gathered}
\frac{\partial(c \phi)}{\partial T}=\frac{\partial c}{\partial X}+\frac{\partial \phi}{\partial T} \\
\frac{\partial \phi}{\partial T}=(1-\phi) c .
\end{gathered}
$$

Numerical integration of Eqs. (5), under suitable initial or boundary conditions, for the evaluation of the functions $\phi$ and $c$ are included in [2]. We must point out here that the estimation of the quantities $\phi$ and $c$ with respect to the true parameters $x$ and $t$ demands the calculation of the quantity $q$. This can be achieved by using the well-known Darcy-law concerning flow in inhomogeneous media. The above calculation procedure is completely developed in [4].

Aim of the present paper is the effort to construct the general solutions of the nonlinear PDEs (5), in the sense that a full complement of arbitrary functions is introduced. These functions can be determined by using convenient initial and boundary conditions. Two kinds of possible boundary conditions are valid at the distant boundary $X=L$ : (i) A condition of the Newmann type, that is $\partial c(L, T) / \partial X=0$, which practically leads to solutions of the form $c=c(T), \phi=\phi(T)$, and (ii) A boundary of the Dirichlet type, that is $c(L, T)=c_{0}=$ constant. On the other hand, the initial conditions of the problem under consideration can be considered as follows:

$$
\phi(X, 0)=\phi(X)=\text { known } \text { and } c(X, 0)=c(X)=\text { known },
$$

or

$$
\phi(X, 0)=\phi_{0}=\text { constant } \text { and } c(X, 0)=c(X)=\text { known }
$$

or

$$
\phi(X, 0)=\phi(X)=\text { known } \quad \text { and } \quad c(X, 0)=c_{0}=\text { constant }
$$

or, finally,

$$
\phi(X, 0)=\phi_{0}=\text { constant } \text { and } c(X, 0)=c_{0}=\text { constant }
$$

\section{GENERAL SOLUTIONS}

The nonlinear system (5) can be rewritten in the form

$$
\begin{aligned}
(c \phi)_{T} & =c_{, T} \phi+c \phi,_{T}=c_{, X}+\phi_{, T}, \\
\phi_{, T} & =(1-\phi) c,
\end{aligned}
$$


where the subscript after the comma indicates partial derivative with respect to this index. Solving Eq. (9) for $c$, evaluating the derivatives $c_{, T}$ and $c_{, X}$ and introducing the results into (8), we derive the following second-order nonlinear PDE for the porosity $\phi$ of the Monge type (Forsyth [5]; Ames [6])

$$
\begin{aligned}
& \phi \phi_{, T T}-\phi_{, T X}=-\frac{1}{1-\phi} \phi_{, T}^{2}+\frac{1}{1-\phi} \phi_{, T} \phi_{, X}+(1-\phi) \phi_{. T} \\
& 0<\phi<1
\end{aligned}
$$

For convenience, we replace $\phi$ by $u=1-\phi$ in (10) and we deduce the equation

$$
\begin{aligned}
& u_{, X T}-(1-u) u_{, T T}+u u_{, T}-\frac{1}{u} u_{, X} u_{, T}+\frac{1}{u} u_{, T}^{2}=0, \\
& 0<u<1
\end{aligned}
$$

which, can be rewritten in the integrated form

$$
\left(\frac{u_{, X}}{u}\right)_{, T}+u_{, T}-\left(\frac{u_{, T}}{u}\right)_{, T}+\left(u_{, T}\right)_{, T}=0 .
$$

Thus, an intermediate integral of the nonlinear PDE (12) becomes

$$
\begin{aligned}
& \frac{u_{, X}}{u}+u-\frac{u_{. T}}{u}+u_{, T}=F(X), \\
& F(X)=\text { arbitrary. }
\end{aligned}
$$

Since $0<u=1-\phi<1$, Eq. (13) results in

$$
(\ln u)_{, X}+u-(\ln u)_{. T}+u_{, T}=F(X) .
$$

By the first functional transformation

$$
\ln u(T, X)=z(T, X) \Rightarrow u=\exp (z)
$$

we succeed in reducing Eq. (14) in the form

$$
\left(z-\int F(X) \mathrm{d} X\right)_{, X}+\mathrm{e}^{z}-z_{, T}+\left(\mathrm{e}^{z}\right)_{, T}=0,
$$

which, further, by the new functional transformation

$$
z-\int F(X) \mathrm{d} X=\omega(T, X) \Rightarrow \mathrm{e}^{z}=\mathrm{e}^{\omega} \mathrm{e}^{\int F(X) \mathrm{d} X},
$$

is transformed to the following quasi-linear equation

$$
\omega_{, X}+\left(\mathrm{e}^{(i)} \mathrm{e}^{\int F(X) \mathrm{d} X}-1\right) \omega_{, T}=-\mathrm{e}^{\omega} \mathrm{e}^{\int F(X) \mathrm{d} X} .
$$


Using the corresponding to Eq. (18) subsidiary Lagrange equations (Ames [6]), that is

$$
\frac{\mathrm{d} X}{1}=\frac{\mathrm{d} T}{\mathrm{e}^{\omega} \mathrm{e}^{\int F(X) \mathrm{d} X}-1}=\frac{\mathrm{d} \omega}{-\mathrm{e}^{\omega} \mathrm{e}^{\int F(X) \mathrm{d} X}}=\frac{\mathrm{d} T+\mathrm{d} \omega}{-1},
$$

and combining the first and third of them, after integration, we deduce

$$
\mathrm{e}^{-\omega}-\int \mathrm{e}^{\int F(X) \mathrm{d} X} \mathrm{~d} X=\alpha=\text { constant }
$$

Also, combination of the first and fourth of the above equations after integration results in

$$
X+T+\omega=\beta=\text { constant }
$$

Recalling the transformation (17) and (15), as well as that $u=1-\varphi$, both last equations became

$$
\begin{aligned}
& \frac{1}{1-\phi} \mathrm{e}^{\int F(X) \mathrm{d} X}-\int \mathrm{e}^{\int F(X) \mathrm{d} X} \mathrm{~d} X=\alpha, \\
& X+T+\ln (1-\phi)-\int F(X) \mathrm{d} X=\beta .
\end{aligned}
$$

Since in Eq. (22) $\alpha$ and $\beta$ are arbitrary constants, the general integral of the porosity equation (10) can be written as follows (Forsyth [5], Ames [6])

$$
G\left(\frac{1}{1-\phi} \mathrm{e}^{\int F(X) \mathrm{d} X}-\int \mathrm{e}^{\int F(X) \mathrm{d} X} \mathrm{~d} X, \quad X+T+\ln (1-\phi)-\int F(X) \mathrm{d} X\right)=0,
$$

or equivalently

$$
\frac{1}{1-\phi} \mathrm{e}^{\int F(X) \mathrm{d} X}-\int \mathrm{e}^{\int F(X) \mathrm{d} X} \mathrm{~d} X=H\left[X+T+\ln (1-\phi)-\int F(X) \mathrm{d} X\right]
$$

where $F$ and $G$, or $F$ and $H$, are arbitrary functions of their arguments.

The proof that Eq. (23) is a general solution of Eq. (10), is given through Eq. (19) and the fact that $\phi=I-u$.

The expression (23), or equivalently (24), constitutes the general solution of the dynamic nonlinear PDE (10) of the porosity, in the sense that a full complement of arbitrary functions $F, G$ or $F, H$, as many as the order of the PDE, is introduced. Here we must point out that only special cases of nonlinear PDEs of the form $f\left(x, y, z_{, x}, z_{, y}, z_{x x}, z_{, x y}, z_{y y}\right)=0$ have been integrated giving general solutions. A few of them expressing general and important geometrical and physical problems have been tabulated in the classical books by Forsyth [5] and Ames [6]. Consequently, as Eq. (10) governs a physical problem of very important interest, the authors believe that it can be classified among the twenty three nonlinear PDEs of the Monge type in physics, for which general solutions have been constructed (Ames [6], pp. 66-68). 
In the followings we shall try to specify the above solution according to the demanded initial conditions of each problem under consideration. For this purpose, we prefer to use the type of the general solution given by Eq. (24), in which one sets

$$
\int \mathrm{e}^{\int F(X) \mathrm{d} x} \mathrm{~d} X=Q(X), \quad e^{\int F(x) \mathrm{d} x}=Q^{\prime}(X), \quad F(X)=\ln Q^{\prime}(X) .
$$

Then the solution (24) can be written under the form

$$
X+T+\ln (1-\phi)-\ln Q^{\prime}=H\left(\frac{Q^{\prime}}{1-\phi}-Q\right) ; \quad \phi=\phi(X, T),
$$

where $Q(X)$ and $H\left(Q^{\prime} /(1-\phi)-Q\right)$ are arbitrary functions of the arguments $X$ and $\left(Q^{\prime} /(1-\phi)-Q\right)$, while prime means derivative with respect to $X$.

\section{INITIAL VALUE PROBLEM}

The problem is focused on the determination of the arbitrary functions $F(X)$ and $H(n)$, $\left[n=X+T+\ln (1-\phi)-\int F(X) \mathrm{d} X\right]$, which are introduced in the general solution (24) under specific initial and boundary conditions. For this purpose the equations which will be used are the following:

$$
\begin{aligned}
\phi_{, T} & =(1-\phi) c, \\
-\frac{\phi_{, X}}{1-\phi}+1-\phi+\frac{\phi_{. T}}{1-\phi}-\phi_{, T} & =F(X)
\end{aligned}
$$

as well as the general solution in the parametric form (22), that is

$$
\begin{aligned}
& \frac{1}{1-\phi} \mathrm{e}^{\int F(X) \mathrm{d} X}-\int \mathrm{e}^{\int F(X) \mathrm{d} X} \mathrm{~d} X=\alpha, \\
& X+T+\ln (1-\phi)-\int F(X) \mathrm{d} X=\beta .
\end{aligned}
$$

Since we have already set

$$
\int \mathrm{e}^{\int F(X) \mathrm{d} x} \mathrm{~d} X=Q(X)
$$

the parametric Eq. (28) can be written as

$$
\begin{array}{r}
\frac{1}{1-\phi} Q^{\prime}(X)-Q(X)=\alpha, \\
X+T+\ln (1-\phi)-\ln Q^{\prime}(X)=\beta .
\end{array}
$$

Now, we will try different types of initial conditions from which we will deduct useful conclusions. 


\section{1st Set of Initial Conditions}

We introduce the initial conditions

$$
\text { For } T=0, \phi=\phi_{0}=\text { constant }, \quad c=c_{o}=\text { constant } \text {. }
$$

Thus, from Eqs. (25) and (27) we compute

$$
\begin{aligned}
F(x) & =1-\phi_{0}+\phi_{0} c_{0}, \\
\ln Q^{\prime}(X) & =\int F(X) \mathrm{d} X=\left(1-\phi_{0}+\phi_{0} c_{0}\right) X, \\
Q^{\prime}(X) & =\mathrm{e}^{\int F(X) \mathrm{d} X}=\mathrm{e}^{\left(1-\phi_{0}+\phi_{0} c_{0}\right) X}, \\
Q(X) & =\int \mathrm{e}^{\int F(X) \mathrm{d} X} \mathrm{~d} X=\frac{1}{1-\phi_{0}+\phi_{0} c_{0}} \mathrm{e}^{\left(1-\phi_{0}+\phi_{0} c_{0}\right) X} .
\end{aligned}
$$

Thus, the general parametric solution (29) becomes

$$
\begin{array}{r}
\left(\frac{1}{1-\phi}-\frac{1}{1-\phi_{0}+\phi_{0} c_{0}}\right) \mathrm{e}^{\left(1-\phi_{0}+\phi_{0} c_{0}\right) X}=\alpha, \\
T+\ln (1-\phi)+\phi_{0}\left(1-c_{0}\right) X=\beta .
\end{array}
$$

or, according to Eq. (24),

$$
\left(\frac{1}{1-\phi}-\frac{1}{1-\phi_{0}+\phi_{0} c_{0}}\right) \mathrm{e}^{\left(1-\phi_{0}+\phi_{0} c_{0}\right) X}=H\left(T+\ln (1-\phi)+\phi_{0}\left(1-c_{0}\right) X\right) .
$$

We ask now for the determination of function $H$ under the specific initial conditions (30). In other words we ask for an integral surface (33), which must pass through the curve

$$
T=0, \phi=\phi_{0}, \quad X=s,
$$

where $s$ is a parameter. The parametric equations (32) become

$$
\begin{array}{r}
\left(\frac{1}{1-\phi}-\frac{1}{1-\phi_{0}+\phi_{0} c_{0}}\right) \mathrm{e}^{\left(1-\phi_{0}+\phi_{0} c_{0}\right) s}=\alpha, \\
\ln (1-\phi)+\phi_{0}\left(1-c_{0}\right) s=\beta,
\end{array}
$$

from which we can eliminate the parameter $s$.

This procedure leads to the equation

$$
s=\frac{1}{1-\phi_{0}+\phi_{0} c_{0}} \ln \alpha-\frac{1}{1-\phi_{0}+\phi_{0} c_{0}} \ln \frac{\phi_{0} c_{0}}{1-\phi_{0}+\phi_{0} c_{0}} .
$$


By now, introducing (36) into the second of Eqs. (35), we find an equation connecting the parameters $\alpha$ and $\beta$, that is

$$
\begin{aligned}
\ln \left(1-\phi_{0}\right) & +\frac{\phi_{0}\left(1-c_{0}\right)}{1-\phi_{0}+\phi_{0} c_{0}} \ln \alpha \\
& -\frac{\phi_{0}\left(1-c_{0}\right)}{1-\phi_{0}+\phi_{0} c_{0}} \ln \frac{\phi_{0} c_{0}}{1-\phi_{0}+\phi_{0} c_{0}}=\beta
\end{aligned}
$$

Eq. (37) is sufficient for the construction of the solution of the specific problem under consideration. In fact introducing the parameters $\alpha$ and $\beta$ from Eq. (35) into Eq. (37), we extract the solution

$$
\begin{aligned}
& \ln \left(1-\phi_{0}\right)+\frac{\phi_{0}\left(1-c_{0}\right)}{1-\phi_{0}+\phi_{0} c_{0}} \ln \left(\frac{1}{1-\phi}-\frac{1}{1-\phi_{0}+\phi_{0} c_{0}}\right) \\
& -\frac{\phi_{0}\left(1-c_{0}\right)}{1-\phi_{0}+\phi_{0} c_{0}} \ln \frac{\phi_{0} c_{0}}{\left(1-\phi_{0}+\phi_{0} c_{0}\right)\left(1-\phi_{0}\right)}=T+\ln (1-\phi)
\end{aligned}
$$

One can observe that the variable $X$ is eliminated. In other words, in this case of the initial conditions (30) the porosity $\phi$ becomes a function of one variable, $T(\phi=\phi(T))$. This solution is of Newmann type and it is not admissible.

The most general set of initial conditions that can be introduced is:

\section{2nd Set of Initial Conditions}

For $T=0, \phi=f(X)=$ known, $\quad c=g(X)=$ known.

Again from (25) and (27) we deduce

$$
\begin{gathered}
\phi_{. T}(X)=(1-f) g, \\
F(X)=1-f+f g-\frac{f^{\prime}}{1-f}, \\
\ln Q^{\prime}=\int F(X) \mathrm{d} X=\ln (1-f)+p(X), p(X)=\int(1-f+f g) \mathrm{d} X, \\
Q^{\prime}=\mathrm{e}^{\int F(X) \mathrm{d} X}=(1-f) \mathrm{e}^{p(X)}, \\
Q=\int \mathrm{e}^{\int F(X) \mathrm{d} X}=\int(1-f) \mathrm{e}^{p(X)} \mathrm{d} X=q(X) .
\end{gathered}
$$


Thus, the general parametric solution (29) becomes

$$
\begin{array}{r}
\frac{1}{1-\phi}(1-f) \mathrm{e}^{p(X)}-q(X)=\alpha, \\
X+T+\ln (1-\phi)-\ln (1-f)-p(X)=\beta .
\end{array}
$$

We ask for the integral surface (41), which must pass through the curve

$$
T=0, \quad \phi=f(X), \quad X=s .
$$

Using the parameter $X=s$, the parametric Eqs. (41) take the form

$$
\begin{array}{r}
\mathrm{e}^{p(s)}-q(s)=\alpha \\
s-p(s)=\beta,
\end{array}
$$

from which we can eliminate the parameter $s$. Indeed, solving the second of these for $s$ we estimate

$$
s=P(\beta)
$$

while, introducing this result into the first of the above equation, we get

$$
\mathrm{e}^{p[P(\beta)]}-q[P(\beta)]=\alpha
$$

Introducing now the expressions of the parameters $\alpha$ and $\beta$ from Eq. (41) into the last equation, we extract the desired solution, that is

$$
\begin{aligned}
& \mathrm{e}^{p[P(X+T+\ln (1-\phi)-\ln (1-f)-p(X))]}-q^{[P(X+T+\ln (1-\phi)-\ln (1-f)-p(X))]} \\
& \quad=\frac{1-f}{1-\phi} \mathrm{e}^{p(X)}-q(X),
\end{aligned}
$$

which involves two independent variables $X$ and $T$. This solution is admissible.

\section{APPLICATIONS AND NUMERICAL RESULTS}

We will present an application by specifing the 2nd set of initial conditions (39) setting

$$
T=0, \phi=f(X)=\mathrm{e}^{-X}, \quad c=c_{0} .
$$

We compute

$$
\begin{gathered}
\phi_{, T}=\left(1-\mathrm{e}^{-X}\right) c_{0}, \\
F(X)=1-\left(1-c_{0}\right) \mathrm{e}^{-X}+\frac{\mathrm{e}^{-X}}{1-\mathrm{e}^{-X}},
\end{gathered}
$$




$$
\begin{gathered}
\ln Q^{\prime}=\int F(X) \mathrm{d} X=p(X)+\ln \left(1-\mathrm{e}^{-X}\right), \quad P(X)=X+\left(1-c_{0}\right) \mathrm{e}^{-X}, \\
Q^{\prime}=\mathrm{e}^{\int F(X) \mathrm{d} X}=\left(1-\mathrm{e}^{-X}\right) \mathrm{e}^{-X+\left(1-c_{0}\right) \mathrm{e}^{-X}} \\
Q=\int \mathrm{e}^{\int F(X) \mathrm{d} X} \mathrm{~d} X=\int\left(1-\mathrm{e}^{-X}\right) \mathrm{e}^{X+\left(1-c_{0}\right) \mathrm{e}^{-X}} \mathrm{~d} X
\end{gathered}
$$

For the determination of the function $Q(x)$ we have the following integrals:

$$
\text { a. } \int \mathrm{e}^{-X+\left(1-c_{0}\right) \mathrm{e}^{-X}} \mathrm{~d} X
$$

and

$$
\text { b. }-\int \mathrm{e}^{\left(1-c_{0}\right) \mathrm{e}^{-X}} \mathrm{~d} X \text {. }
$$

Setting

$$
\mathrm{e}^{-X}=t, \quad-\mathrm{e}^{-X} \mathrm{~d} X=\mathrm{d} t, \quad \mathrm{~d} X=-\mathrm{e}^{-X} \mathrm{~d} t=-\frac{\mathrm{d} t}{t}
$$

we have (see Ref. [7], p. 93)

$$
\text { a. }-\int \frac{1}{t} \mathrm{e}^{\left(1-c_{0}\right) t} \frac{\mathrm{d} t}{t}=-\int \frac{\mathrm{e}^{\left(1-c_{0}\right) t}}{t^{2}} \mathrm{~d} t=\frac{\mathrm{e}^{\left(1-c_{0}\right) t}}{t}-\left(1-c_{0}\right) E_{i}\left[\left(1-c_{0}\right) t\right],
$$

and

$$
\text { b. } \int \frac{\mathrm{e}^{\left(1-c_{0}\right) t} \mathrm{~d} t}{t}=E_{i}\left[\left(1-c_{0}\right) t\right]
$$

where $E_{i}(t)$ is the exponential integral function, $E_{i}(t)=-\lim \left[\int_{-s}^{-\varepsilon} \mathrm{e}^{-s} / s \mathrm{~d} s+\int_{\varepsilon}^{\infty} \mathrm{e}^{-s} / s \mathrm{~d} s\right]$, $t>0$ (see Ref. [7], p. 925).

Thus, $Q(x)$ is estimated by the formula

$$
Q(X)=\frac{\mathrm{e}^{\left(1-c_{0}\right) \mathrm{e}^{-X}}}{\mathrm{e}^{-X}}+c_{0} E_{i}\left[\left(1-c_{0}\right) \mathrm{e}^{-X}\right]
$$

The general parametric solutions (29) according to (48) and (49), become

$$
\begin{aligned}
& \frac{1}{1-\phi}\left(1-\mathrm{e}^{-X}\right) \mathrm{e}^{X+\left(1-c_{0}\right) \mathrm{e}^{-X}}-\mathrm{e}^{X+\left(1-c_{0}\right) \mathrm{e}^{-X}}-c_{0} E_{i}\left[\left(1-c_{0}\right) \mathrm{e}^{-X}\right]=\alpha, \\
& T+\ln \left(\frac{1+\phi}{1-\mathrm{e}^{-X}}\right)-\left(1-c_{0}\right) \mathrm{e}^{-X}=\beta .
\end{aligned}
$$


We ask for the integral surface, which must pass through the curve

$$
T=0, \quad \phi=\mathrm{e}^{-X}, \quad X=s
$$

where $s$ is a parameter.

In this case Eqs. (50) result in

$$
\begin{aligned}
c_{0} E_{i}\left[\left(1-c_{0}\right) \mathrm{e}^{-s}\right] & =-\alpha, \\
\left(1-c_{0}\right) \mathrm{e}^{-s} & =-\beta, \quad-\beta>0,
\end{aligned}
$$

from which we eliminate the parameter $s$. Introducing the second of (52) into the first of them, we find

$$
c_{0} E_{i}(-\beta)=-\alpha, \quad-\beta>0 .
$$

Using the asymptotic representation of the exponential integral function $E_{i}(X)$ for $X>0$ (see Ref. [7], p. 925) we have

$$
E_{i}(-\beta)=\mathbf{C}+\ln (-\beta)+\sum_{k=1}^{\infty} \frac{(-\beta)^{k}}{k k !},
$$

where $\mathbf{C}$ is the Euler's constant $=0.5772156649015325 \ldots$

Thus, the desired solution (53) becomes according to Eq. (50)

$$
\begin{aligned}
c_{0}\{\mathbf{C}+ & \ln \left[-T+\ln \left((1-\phi) /\left(1-\mathrm{e}^{-X}\right)\right)-\left(1-c_{0}\right) \mathrm{e}^{-X}\right] \\
& \left.+\sum_{k=1}^{\infty} \frac{\left[-T+\ln \left((1-\phi) /\left(1-\mathrm{e}^{-X}\right)\right)-\left(1-c_{0}\right) \mathrm{e}^{-X}\right]^{k}}{k k !}\right\} \\
= & \frac{1}{1-\phi}\left(1-\mathrm{e}^{-X}\right) \mathrm{e}^{X+\left(1-c_{0}\right) \mathrm{e}^{-X}}+\mathrm{e}^{X+\left(1-c_{0}\right) \mathrm{e}^{-X}} \\
& +c_{0}\left\{\mathrm{C}+\ln \left[\left(1-c_{0}\right) \mathrm{e}^{-X}\right]+\sum_{k=1}^{\infty} \frac{\left[\left(1-c_{0}\right) \mathrm{e}^{-X}\right]^{k}}{k k !}\right\}
\end{aligned}
$$

\section{NUMERICAL SOLUTION}

From now on, we focus on the numerical solution of Eqs. (38) an (55) concerning the already examined two kinds of initial conditions. We choose the following values of the introduced dimensionless parameters (see Ref. [8]):

- length $L=1,0$;

- Initial porosity $\phi(X, 0)=\phi_{0}=0.25$;

- Initial concentration of transport $c(X, 0)=c_{0}=10^{-3}$;

- Time step $\Delta T=0.1$;

- Space step $\Delta X=0.1$. 
At first we solve numerically Eq. (38), in which the variable $X$ has been eliminated and the porosity $\phi$ is only a function of the variable $T(\phi=\phi(T))$. Figures 1 and 2 present the time evolution of porosity independently of the location, for small and large times respectively. In these two figures one observes that the porosity increases by time reaching the maximum value of $\phi=1,0$. These results are in complete agreement with the results given in Ref. [8].

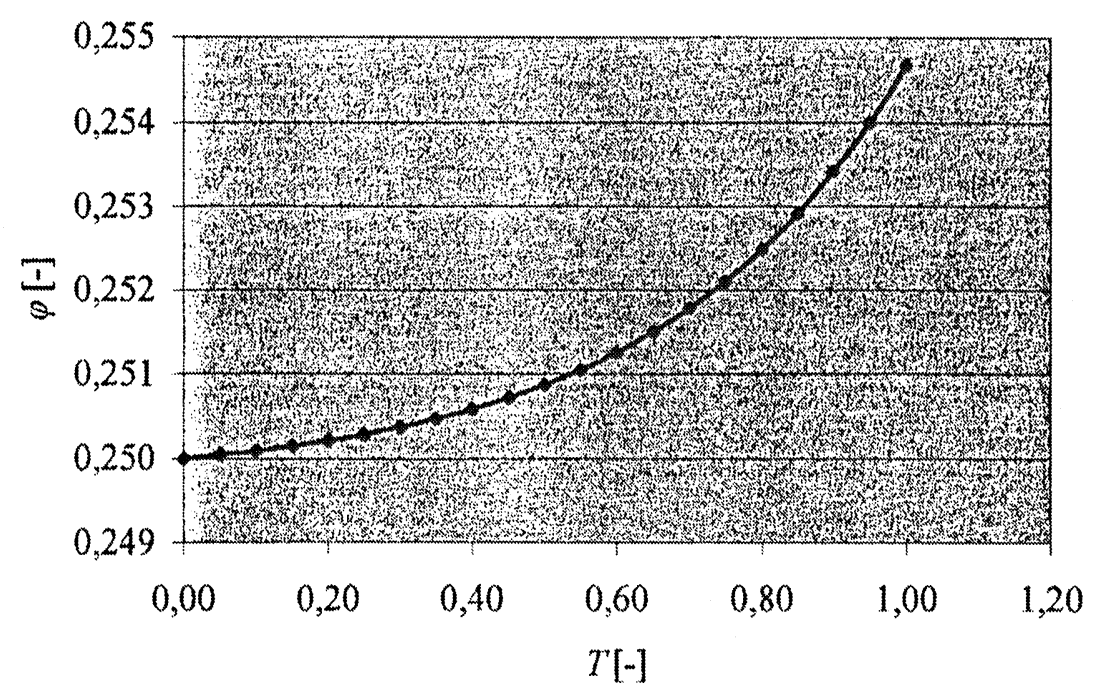

FIGURE 1 Time evolution of porosity (solution independent of $X$ ).

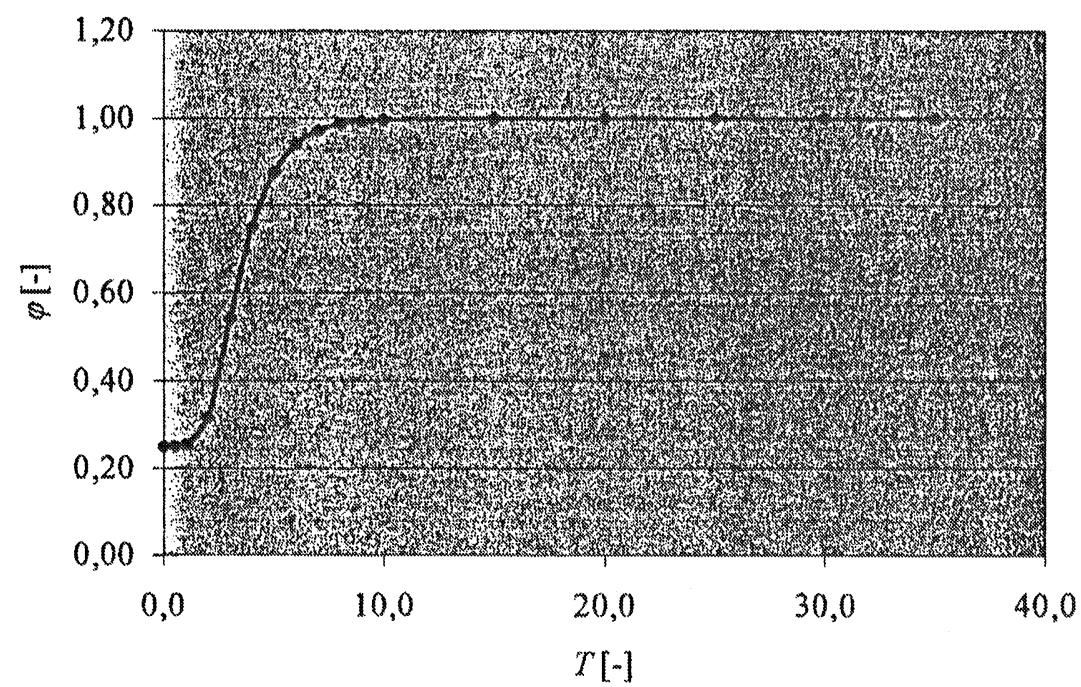

FIGURE 2 Time evolution of porosity (solution independent of $X$ ). 
Secondly we solve numerically Eq. (55). The results are drawn for various values of $X$ and $T$, and the porosity $\phi$ is calculated. In Figures 3 to 7 we present the space evolution of porosity for various times, while in Figure 8 we present Figures 3 to 7 . We observe, that the porosity $\phi$ is reduced as $X$ increases at any time. Furthermore Figures 9 to 12 present the time evolution of porosity at various locations, while Figure 13 presents Figures 9 to 12 . Here, one observes that the porosity increases with the time at any location, and especially, in Figure 13, the porosity is reduced at any location as we distant from the source of the well, but increases as the time increases at any location. This is a fact that proves that the porosity moves like a diffused wave. These results are again in complete agreement with the results given in Ref. [8].

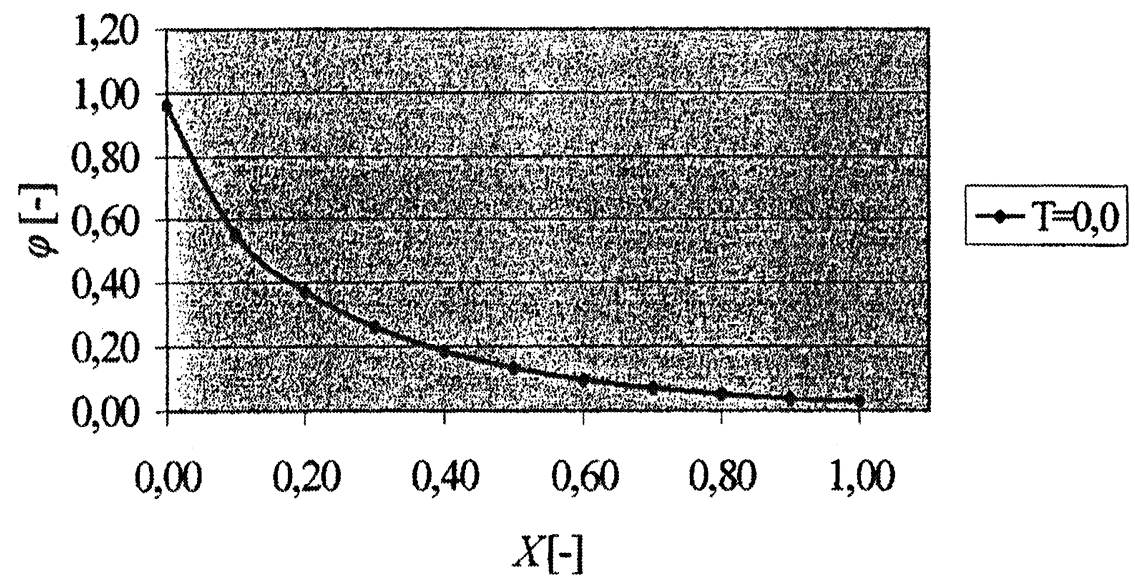

FIGURE 3 Space evolution of porosity for $T=0,0$.

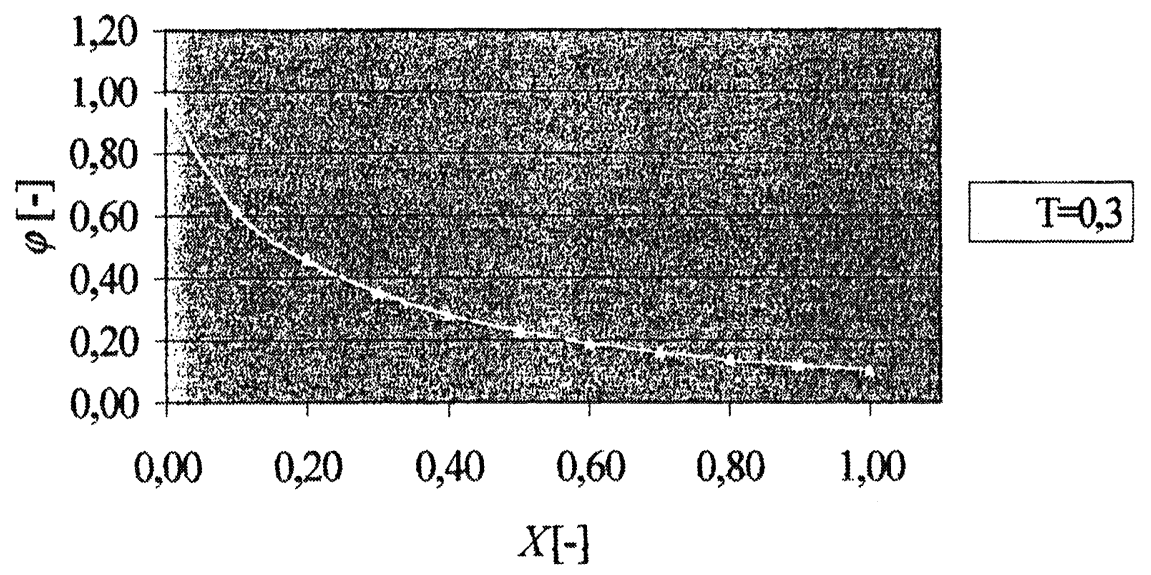

FIGURE 4 Space evolution of porosity for $T=0,3$. 


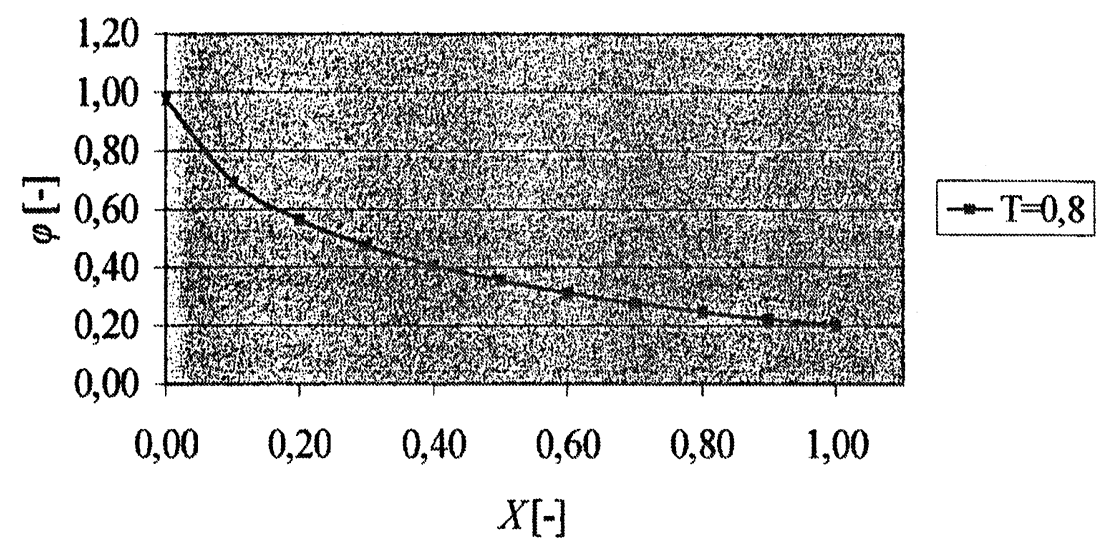

FIGURE 5 Space evolution of porosity for $T=0,8$.

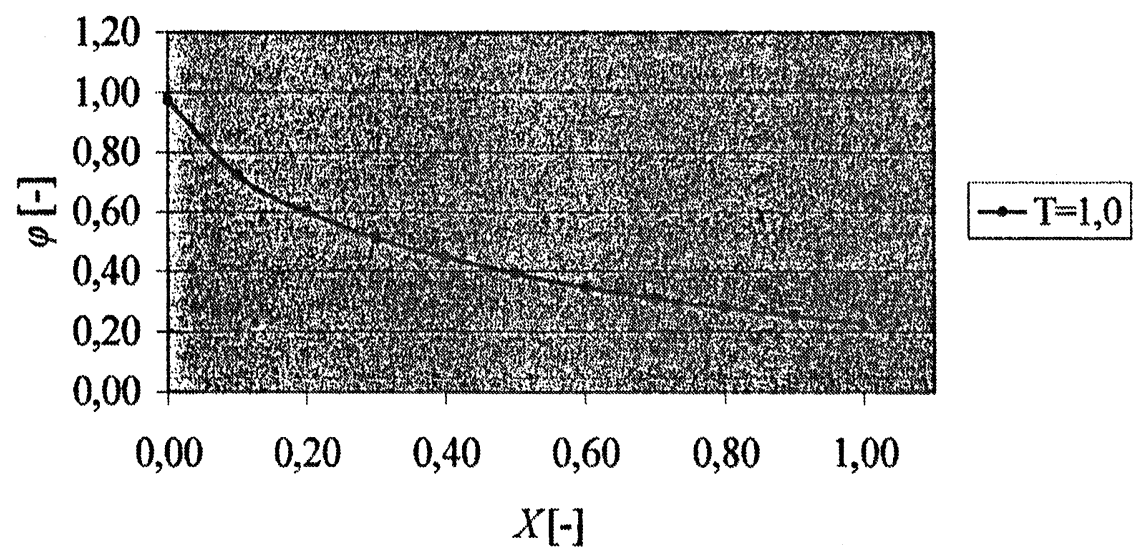

FIGURE 6 Space evolution of porosity for $T=1,0$.

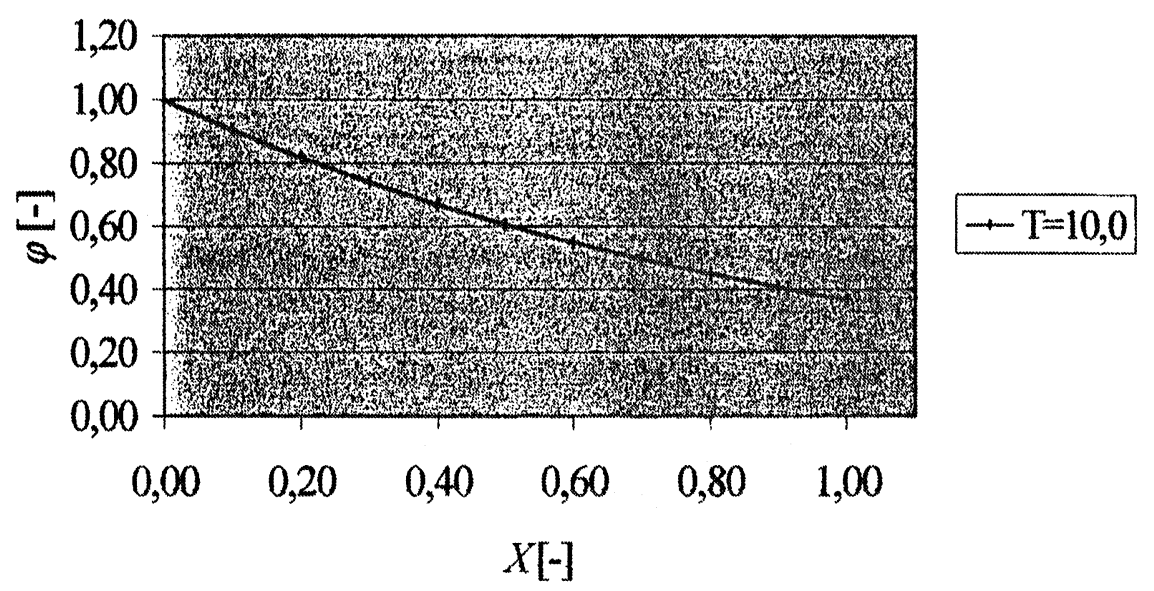

FIGURE 7 Space evolution of porosity for $T=10,0$. 

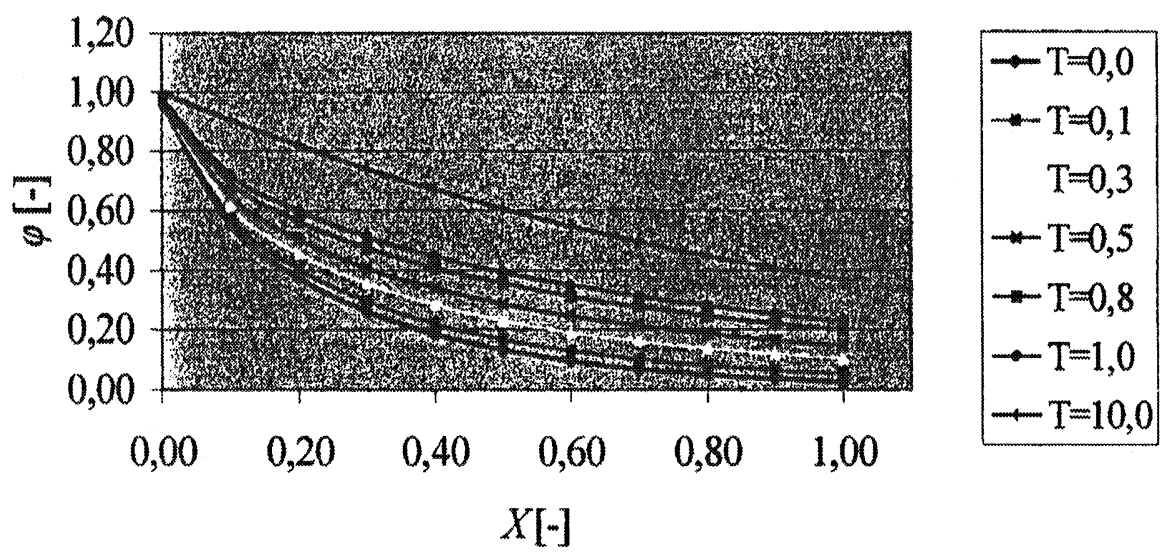

FIGURE 8 Space evolution of porosity for various times.

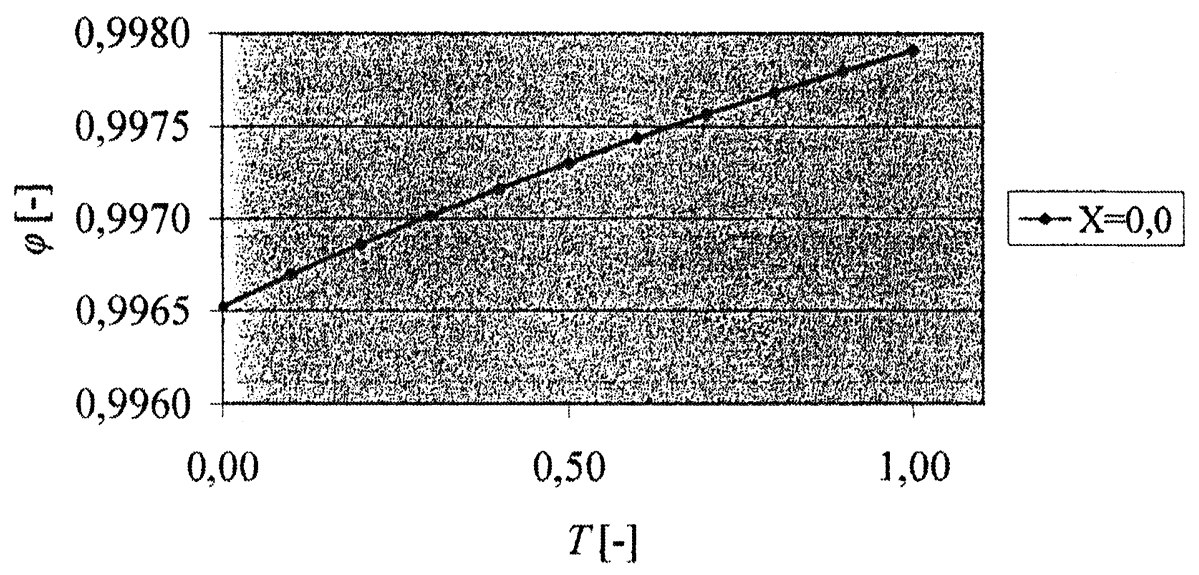

FIGURE 9 Time evolution of porosity at location $X=0,0$.

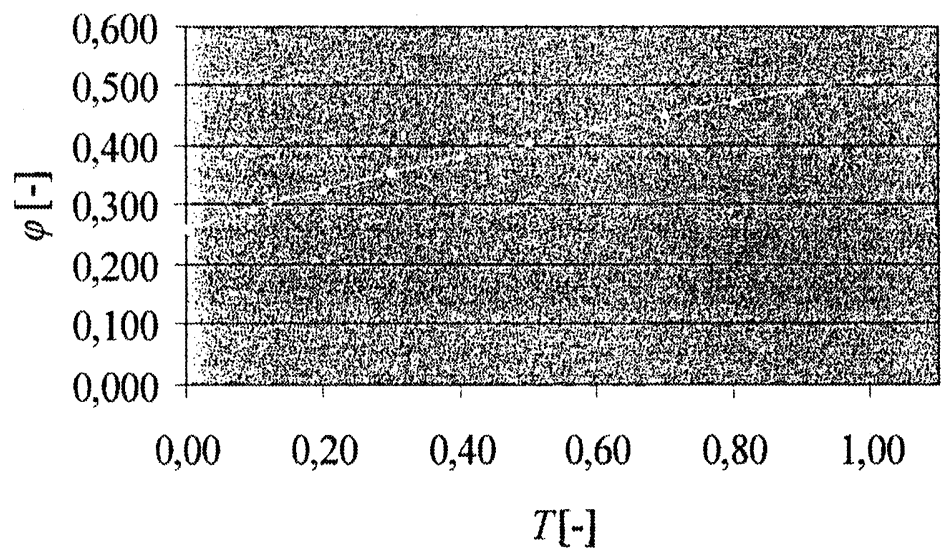

$\mathrm{X}=0,3$

FIGURE 10 Time evolution of porosity at location $X=0,3$. 


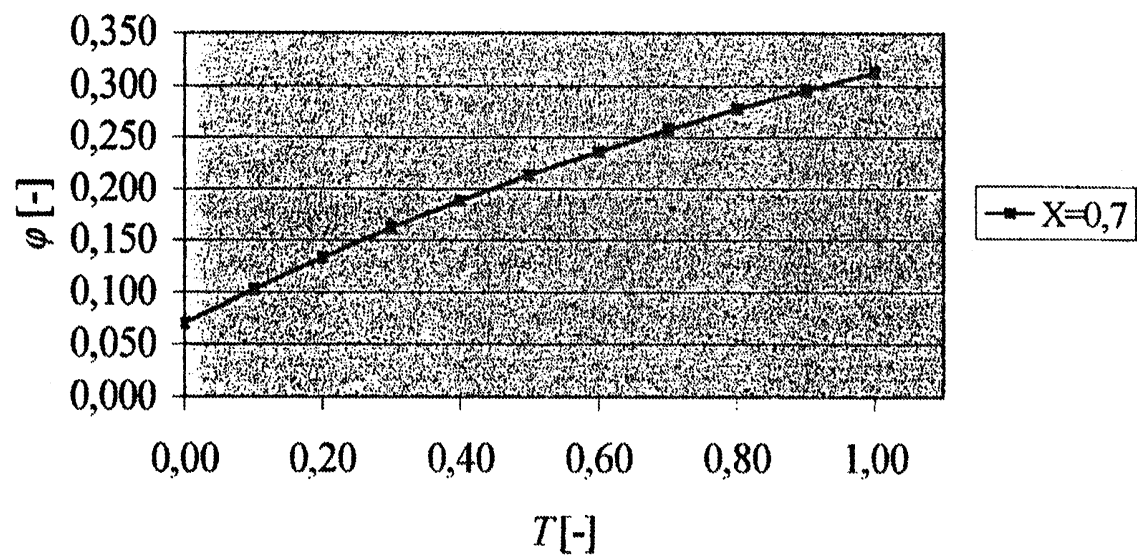

FIGURE 11 Time evolution of porosity at location $X=0,7$.

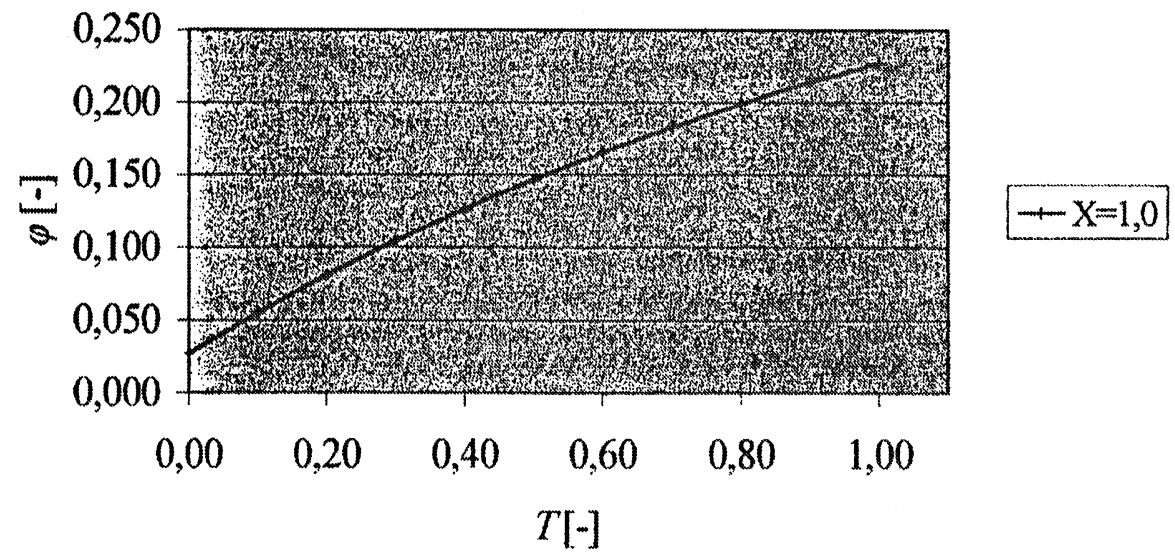

FIGURE 12 Time evolution of porosity at location $X=1,0$.

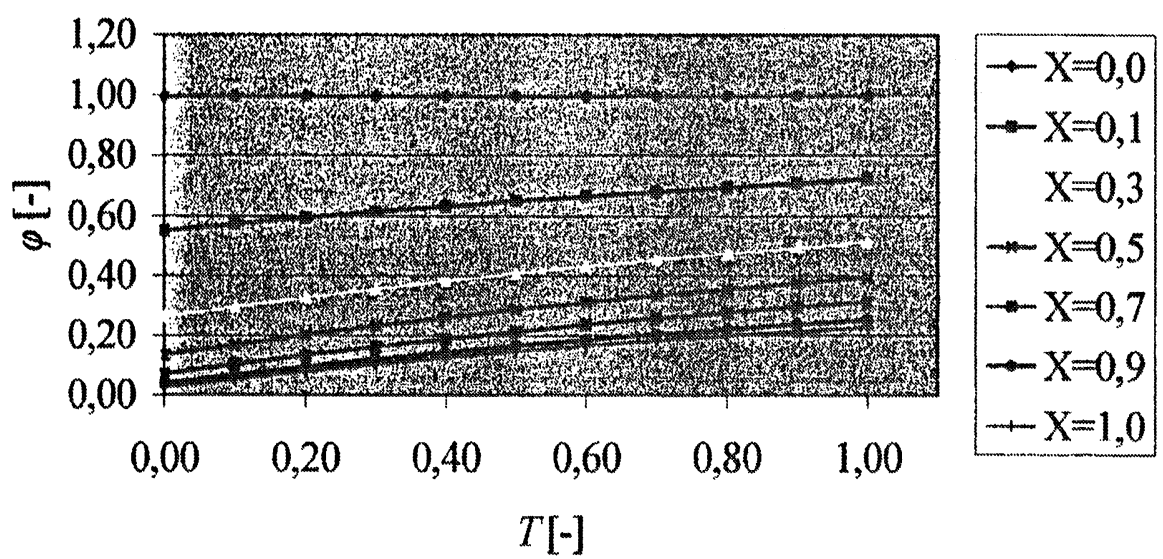

FIGURE 13 Time evolution of porosity at various locations. 


\section{CONCLUSIONS}

We succeed in giving general solutions concerning the nonlinear PDEs which govern the erosion kinetics. It is proved that the constructed second-order nonlinear PDE of the Monge type referring to the porosity can be analytically integrated in the sense that a full complement of arbitrary functions (as many as the order) is introduced. Thus, one can specify the above general solution according to the demanded conditions of each problem under consideration. In the present case this specification takes place through the initial conditions proposed by Vardoulakis, Stavropoulou and Papanastasiou [2] and Stavropoulou [8] and are in complete agreement with their results extracted by the numerical solution of the fundamental PDEs [5].

\section{References}

[1] Veeken, M. G. A., Davies, D. R., Kenter, G. J. and Kooijman, A. P. (1991) Sand Production Review: Developing and Integral Approach (pp. 335-346). SPE22792.

[2] Vardoulakis, I., Stavropoulou, M. and Papanastasiou, P. (1996) Hydro-mechanical aspects of sand production, Transport in Porous Media, 22, 225-244.

[3] Einstein, H. A. (1937) In: Zurich, T. H. (Eidg.), Der Geschiebetrieb als wahrschein - lichkeits Problem, Mitt.d. Versuchsanstalt $f$. Wasserbau.

[4] Sakthivadivel, R. and Irmay, S. (1996) A Review of Filtration Theories, HEL 15-4. Berkeley: University of California.

[5] Forsyth, A. R. (1959) Theory of Differential Equations, Vols. 5 and 6. New York: Dover.

[6] Ames, W. (1965) Nonlinear Partial Differential Equations in Engineering. New York: Academic Press.

[7] Gradshteyn, I. S. and Ryzhik, I. M. (1965) Table of Integrals, Series and Products. New York: Academic Press.

[8] Stavropoulou, M. (1996) Coupled Hydro-mechanical Instabilities in Porous Media, PhD thesis, Greece: NTUA, Athens. 


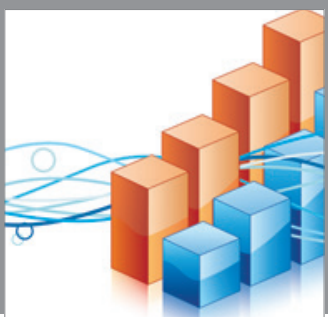

Advances in

Operations Research

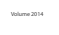

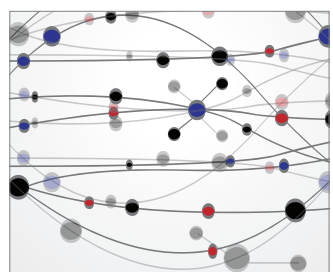

\section{The Scientific} World Journal
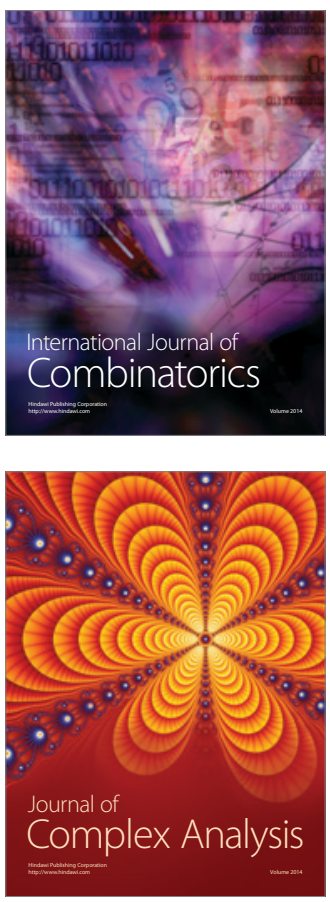

International Journal of

Mathematics and

Mathematical

Sciences
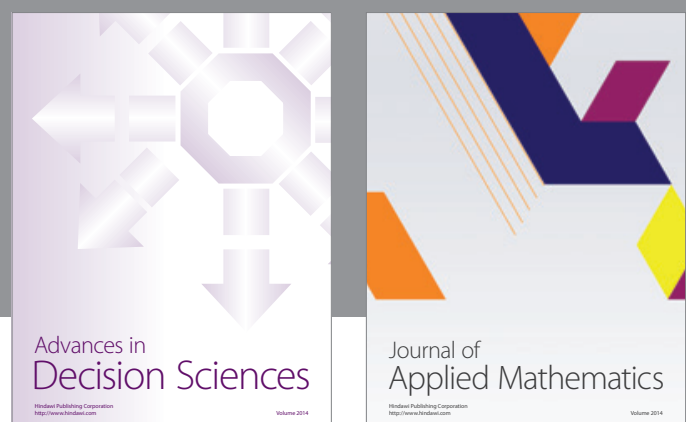

Journal of

Applied Mathematics
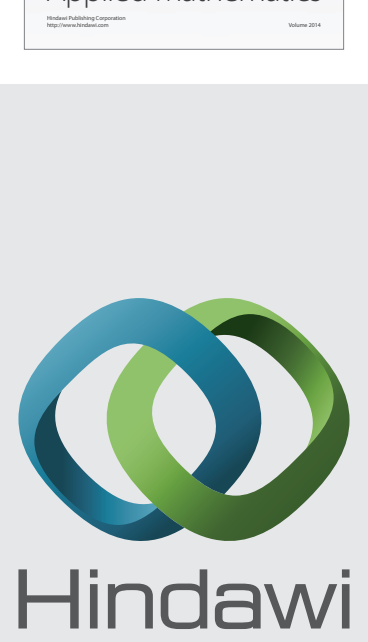

Submit your manuscripts at http://www.hindawi.com
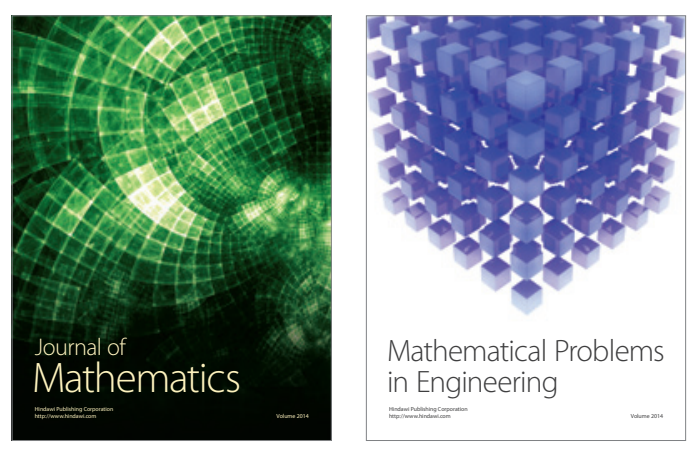

Mathematical Problems in Engineering
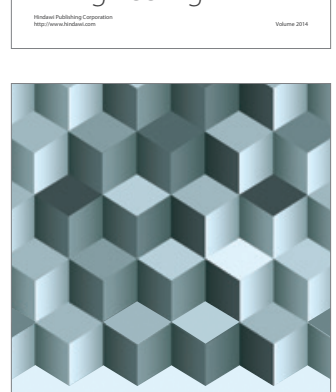

Journal of

Function Spaces
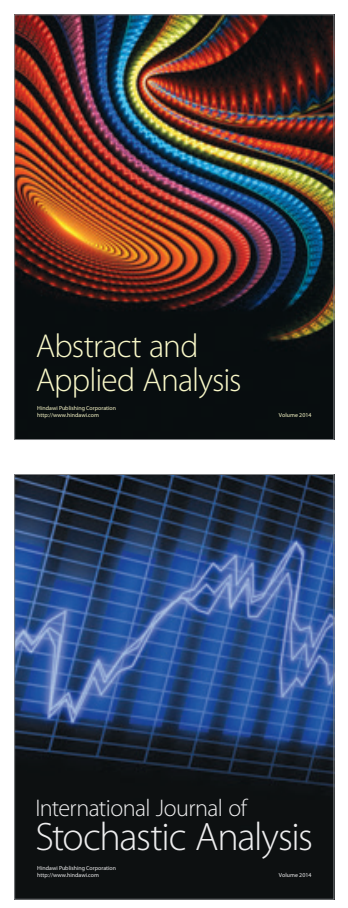

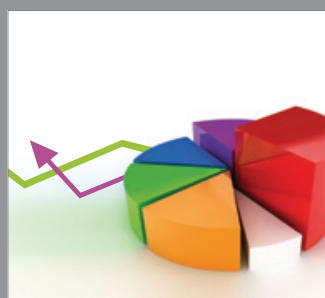

ournal of

Probability and Statistics

Promensencen
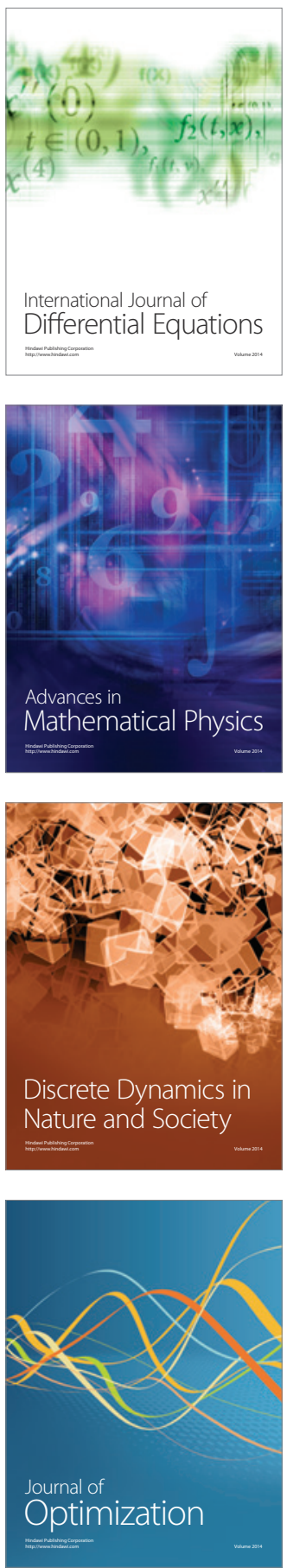